\section{Structure watch}

\section{A DRAMATIC WAY TO REMODEL}

Molecular chaperones, such as heat-shock protein-90 (Hsp90), are ATP-driven molecular machines that facilitate the correct folding of client proteins. New structural studies show how dramatic conformational changes that occur during the Hsp90 reaction cycle might be coupled to clientprotein remodelling.

Hsp90 proteins comprise three domains that are connected by hinge regions, and they form dimers through their C-terminal domains (CTDs). Shiau et al. determined the structure of the Escherichia coli Hsp90 orthologue HtpG in the nucleotide-free, ATP-analogue-bound and ADPbound states. Each of the HtpG states adopts distinct conformations, and the conformational changes induced by nucleotide binding are far more dramatic compared with the previously published findings with yeast Hsp90. The hinge regions between the $\mathrm{N}$-terminal domain (NTD) and the middle domain (MD) as well as between the MD and the CTD are thought to enable this conformational flexibility.

In the nucleotide-free state, the NTD, MD and CTD of the HtpG monomer each present hydrophobic surface areas into a large $\mathrm{V}$-shaped cleft formed by the dimer. The authors propose that this state is probably most optimal for binding client proteins. The addition of ATP leads to changes in the conformation of the NTD and MD. The dimer changes to a more compact ATP-bound state in which the distance between the NTDs decreases and the so-called 'ATP lid' loop structure moves over the bound nucleotide. This more compact configuration is likely to affect client-protein remodelling. Nucleotide hydrolysis results in the very compact ADP-bound state that is expected to release the client protein, thereby resetting the chaperone for binding a new client protein.

The first glimpse of Hsp90 in complex with a client protein was recently provided by Vaughan et al. They expressed and purified a complex of yeast $\mathrm{Hsp} 90$ in complex with the cochaperone $\mathrm{Cdc} 37$ and the client protein $\mathrm{Cdk} 4$, and demonstrated a 2:1:1 ratio for the Hsp90-Cdc37-Cdk4 complex. Taken together with previous data, the authors suggest the formation of an initially symmetric interaction between dimeric $\mathrm{Hsp} 90$ and dimeric $\mathrm{Cdc} 37$ carrying the client protein, after which a $\mathrm{Cdc} 37$ molecule is released. Electronmicroscopy data indicate that $\mathrm{Cdk} 4$ adopts a lobe-shaped extended conformation when bound to the NTD and MD on the outside of one Hsp90 monomer. Cdc37 is thought to be bound to the NTD of the other Hsp90 monomer. The orientation of $\mathrm{Cdk} 4$ relative to $\mathrm{Hsp} 90$ provides a possible mechanism by which ATP-dependent changes in the conformation of the NTD and MD of Hsp90 might be coupled to the remodelling of the client protein.

REFERENCES Shiau, A. K. et al. Structural analysis of E. coli hsp90 reveals dramatic nucleotide-dependent conformational rearrangements. Cell 127, 329-340 (2006)| Vaughan, C. K. et al. Structure of an Hsp90-Cdc37-Cdk4 complex. Mol. Cell 23 697-707 (2006)

FURTHER READING Ali, M. M. U. et al. Crystal structure of an Hsp90-nucleotidep23/Sba1 closed chaperone complex. Nature 440, 1013-1017 (2006)

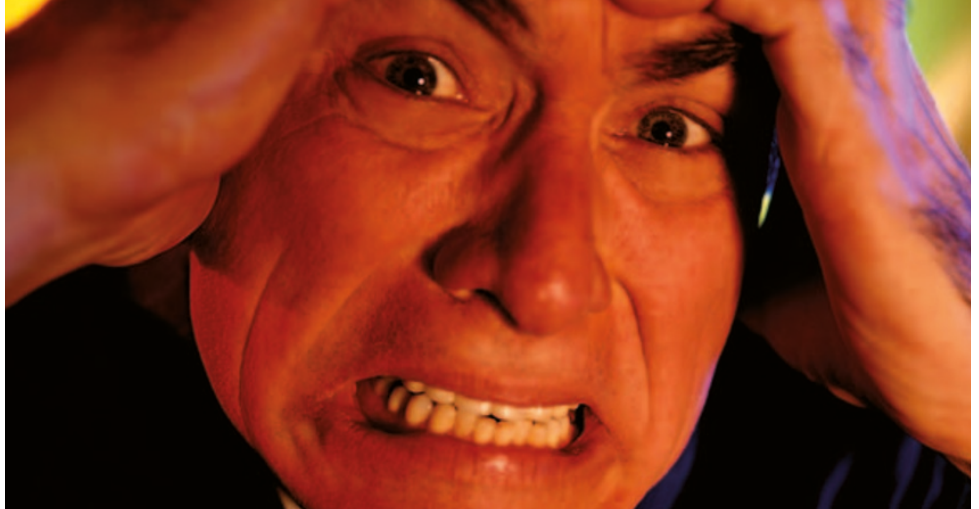

$\Rightarrow$ CELL SIGNALLING

\title{
Even receptors get stressed
}

We all have our own way of dealing with stress - but how do our cells deal with it at a molecular level? Chachisvilis and colleagues now report that mechanical stress across the plasma membrane of endothelial cells can directly activate G-proteincoupled receptors (GPCRs).

It is known that blood pressure can be regulated by shear stress. An increase in blood volume, for example, causes pressure against the endothelial cell lining of blood vessels that activates signalling pathways. These pathways control the level of contraction in the vascular smooth muscle and, in the longer term, contribute to changes in the structure of the vasculature observed during some pathologies. However, the precise mechanisms by which the endothelial cells convert the mechanical stimulus into a biochemical response have been difficult to establish.

Chachisvilis and colleagues studied the $\mathrm{B}_{2}$ bradykinin GPCR that, upon binding to bradykinin, undergoes a conformational change in its structure that activates intracellular heterotrimeric G-proteins. The authors studied the activation of the $B_{2}$ receptor using intramolecular fluorescence resonance energy transfer (FRET).

Chachisvilis and colleagues first confirmed that a $\mathrm{B}_{2}$ 'chameleon' receptor, named because of its colour-changing properties associated with FRET, was correctly expressed at the plasma membrane in cultured endothelial cells. Stimulation of the receptor with the agonist bradykinin caused changes in the FRET signal, due to the movement of helices in the receptor as it switched from its inactive to active conformation. By contrast, application of a receptor antagonist induced no change in the receptor.
These experiments confirm that the chameleon receptor can be used to report GPCR activation.

The authors then stimulated the cells with shear stress by loading the cells in a chamber and altering the flow of fluid across the surface of the cells. The cells were stimulated with two other types of mechanical stress; membrane stretch that was induced by changing the osmolarity of the bathing media and changes in the fluidity of the plasma membrane. All three types of stress caused a conformational change in the receptor, similar to that observed during the activation of the receptor by bradykinin.

Previous studies have been unable to confirm whether mechanical stress can directly activate the receptors or whether the mechanism of activation is indirect by, instead, stimulating the release of autocrine-signalling molecules from the cells. In this study, however, this is unlikely because the chameleon receptors require higher concentrations of agonist than the native receptors to be activated. In addition, an inhibitor that prevents the production of a possible autocrine molecule had no significant effect on receptor activation. Chachisvilis and colleagues are confident therefore that their observed effects of mechanical stress are due to the direct activation of the membrane receptor. If so, this study provides a starting point to understand how factors such as membrane composition and tension might influence the dynamic conformation of GPCRs.

James Pickett

ORIGINAL RESEARCH PAPER Chachisvilis, M. et al. G protein-coupled receptors sense fluid shear stress in endothelial cells. Proc. Natl Acad. Sci. USA 103, 15463-15468 (2006) WEB SITE

Mirianas Chachisvilis' laboratory: http://www.ljbi.org/Chachisvilis_lab.htm 